\title{
THE MIGRATION OF SUNSPOT ACTIVITY ALONG SOLAR MERIDIANS AND PARALLELS
}

\author{
L. Dezsö, O. Gerlei, and ÁGnes Kovács \\ (Heliophysical Observatory of the \\ Hungarian Academy of Sciences, Debrecen, Hungary)
}

\begin{abstract}
A BSTRACT
The places of local maxima of sunspot activity, i.e. the formation of solar active regions, seem to follow in a definite sequence of tracks over the Sun's surface. These sequences of activity apparently represent distinct continuous streams, whose duration may be of the order of magnitude of 1 year.
\end{abstract}

\section{Introduction}

It has been taken always as one of the crucial points in studying the nature and cause of the solar cycle to try to interpret the variation in the average heliographic latitude of sunspots, i.e., the butterfly diagram. But if the butterfly diagram has some fine structure in reality, then any theory concerning the solar cycle that ignores this fact becomes questionable.

We would like to call attention to the peculiarity that the butterfly diagram can be resolved into several tracks of bands with a time-scale of only 1 to 2 years, i.e., considerably less than 11 years. This peculiarity was indicated already by Lockyer (1904), using the data of observations from 1861-1902, and much more clearly by Kuleshova (1962), from Tashkent observations of solar cycles no. 17-19. One may find traces of this property even in one of Bell's papers (1960), who took into account some magnetic measurements too.

Since solar activity may be best characterized through the strengthening of local magnetic fields and the largest field strengths on the Sun are to be found in spot umbrae, it would therefore be most appropriate to investigate the problem on the basis of magnetic measurements relating to umbrae. But since we have no suitable data of this kind available, we used umbra areas, selecting those cases when the umbra of the spot group was growing. We did this because it was highly probable that during each such period the magnetic field was locally also growing.

\section{Data used}

Observations of the heliographic coordinates $(B, L)$ and the daily areal increases of umbrae $(\Delta U>0)$, separately for each sunspot group, were taken from the Greenwich

Kiepenheuer (ed.), Structure and Development of Solar Active Regions, 70-76. C. I.A.U. 
Photo-Heliographic Results for the years 1889-1955, i.e., for 6 entire solar cycles (nos. 13-18). These Greenwich records contain in fact solar observations for every day, but daily only one. We set certain reasonable limits, neglecting certain observations, in order to avoid systematic measuring errors (Dezső, 1964). Thus we confined ourselves to certain values as regards the magnitude of $\Delta U$, the distance from the central meridian $\left(L_{\mathrm{CM}}\right)$ and the lifetimes $(u)$ of the spot groups. When we speak of a spot group of $u$ days, it means that between its first and last (ultimate) observations there is an interval of $u-1$ days. Wherever we used an average value of the heliographic position, this is always an appropriate calculated weighted mean. (We used as weights the number of $\Delta U$ cases and the $\Sigma \Delta U$ values in Sections 3 and 4 respectively.)

While we studied data of observations over a 67 -year period, the present communication gives only a few typical examples* from the solar cycle no. 18 , to illustrate our preliminary results relating to certain migrations of sunspot activity.

\section{Migrations shown by a Special Index of Spot Activity}

Consequent to what was said above, we may introduce quite a practical and reasonable index of sunspot activity $(J)$ by using the number of $\Delta U \geqslant 2$ cases of spot groups. To be quite clear: we counted for each day the number of those spot groups which by the next day has shown an umbra increase of at least 2 areal units $\left(10^{-6}\right.$ of the solar hemisphere), and summed up these numbers for half-year periods, starting from the beginning of the calendar year for every subsequent 3 months. We determined this $J$ index of sunspot activity for areas of $8^{\circ}$ latitude $\times 60^{\circ}$ longitude of the solar surface, starting from the equator $\left(B=0^{\circ}\right)$ and Carrington's zero meridian $\left(L=0^{\circ}\right)$ for every 4th parallel and every 30th meridian. We used observations of a spot group for the calculations of our $J$ indices only when the central-meridian distance of the group was less than $50^{\circ}\left(\left|L_{\mathrm{CM}}\right|<50^{\circ}\right)$, and used a spot group only if its lifetime was at least 5 days $(u \geqslant 5)$.

In the topmost part of Figure 1 a 2-year variation of sunspot activity $(J)$ between Northern latitudes of $20^{\circ}$ and $28^{\circ}$ and longitudes of $60^{\circ}$ and $120^{\circ}$ (i.e., the center of the longitude zone is $90^{\circ}$ ) is shown. We note from the other similar curves of Figure 1 , plotted for the longitude zones of $60^{\circ}, 30^{\circ}, 0^{\circ}$ and $330^{\circ}$, that the $J$-maximum at 1945.5 shifted by $120^{\circ}$ in about a year. Such behaviour, a shift in longitude of the maxima of local solar activity in any zone of latitude, is quite common. Sometimes a much smaller or greater shift occurs. But up to the present we have not been able to establish any definite regularities relating to this shift, anyhow it does not move only towards the decreasing longitudes but equally in the other direction as well.

If we determine the variation of spot activity in different zones of latitude, even by disregarding the distinction of longitudes, it is easy to recognize at once from several

* Several other examples were shown at the Symposium. 


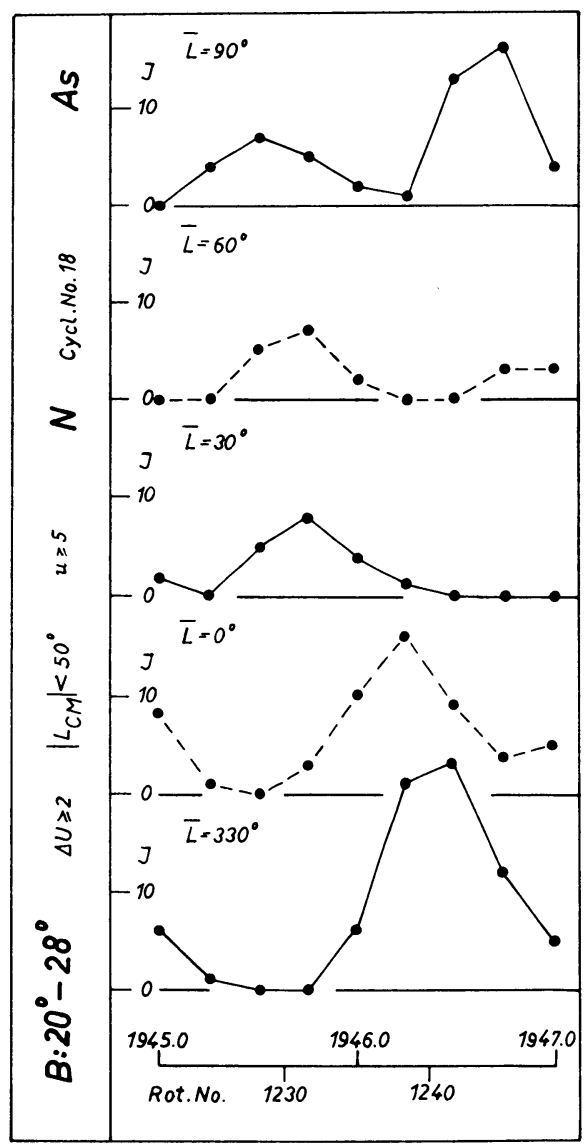

Fig. 1. Variations in sunspot activity on several small areas of the solar surface.

well-defined maxima that the spot activity in general migrates with considerable speed towards lower latitudes. The velocity of these migrations is about one order of magnitude greater than the one which follows from the so-called Spörer's law. We would point out, however, that we noticed amongst the less important migrations of this kind a few which moved toward one of the poles. Nevertheless the dominant characteristics of the latitude migration of sunspot activity is a gradual descent to the lower latitudes. It is perhaps the most important feature of these migrations that before an activity track fades out, not far from the equator, a new one appears at higher latitudes. We may observe at least four main tracks of sunspot activity with fast latitude variation during every solar cycle in each hemisphere. Consequently, one should regard the butterfly diagram as also being built up by such tracks.

In Figure 2 we present examples to illustrate what we have said above. The three curves of sunspot activity relate to the $20^{\circ}-28^{\circ}, 16^{\circ}-24^{\circ}$ and $12^{\circ}-20^{\circ}$ latitude zones. 
A part of the shift for two of the 'main' tracks are to be seen. The times of maxima of local activity and the related average latitudes, indicated according to the scale on the right-hand side of the figure, are shown by crosses.

We think that the curves of Figure 2, and other similar graphs, prove sufficiently well the rapid latitude variation of spot activity. But Figure 3, and some other similar

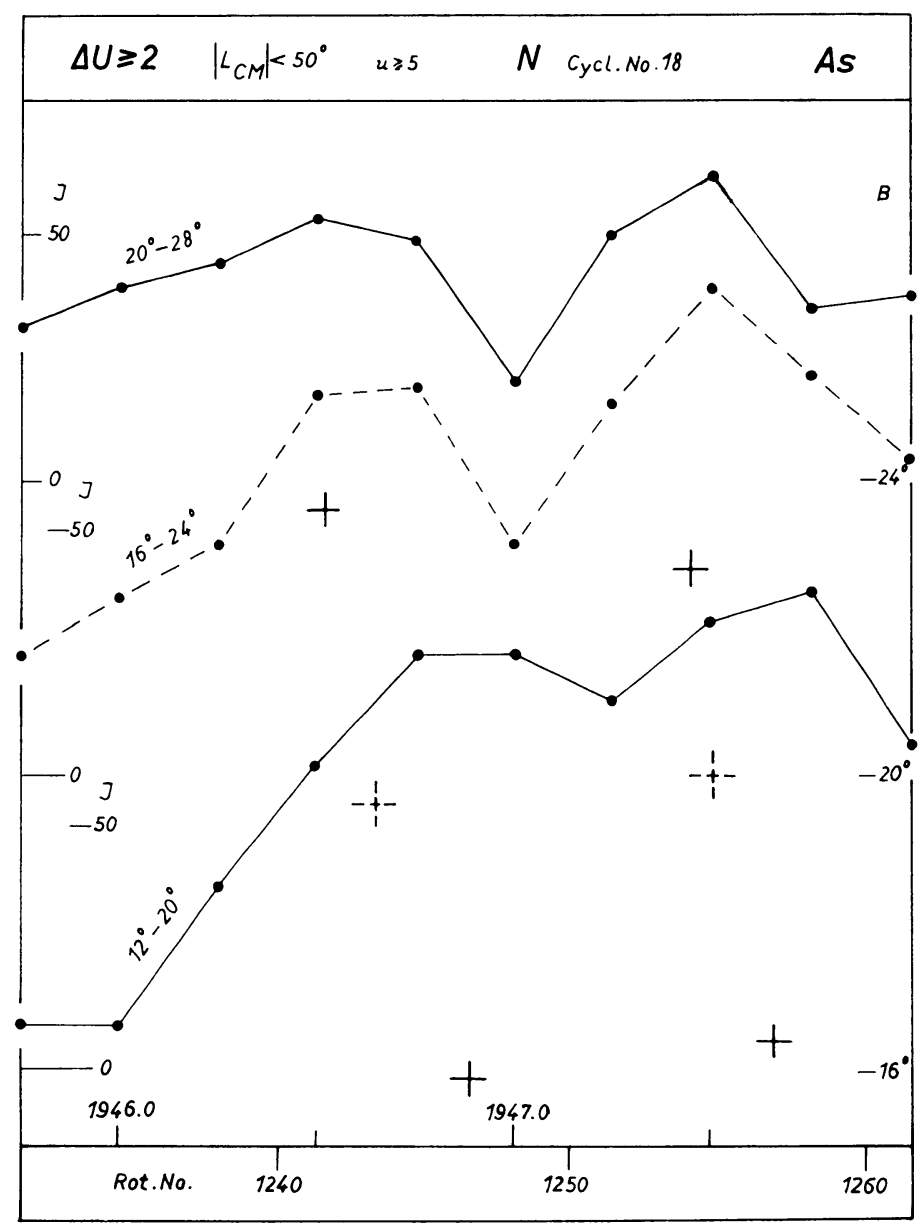

FIG. 2. Variations in sunspot activity in various belts of latitude.

graphs, convince us of it even more. In Figure 3 we show how the spot activity is distributed along longitudes at the times of maxima for the earlier (1946) track of Figure 2. By comparing the three curves of Figure 3, we find that the local maximum has in fact descended to a lower zone of latitude, because during its shift (in 0.34 years) the distribution of activity in longitude on the whole remains the same; or to 


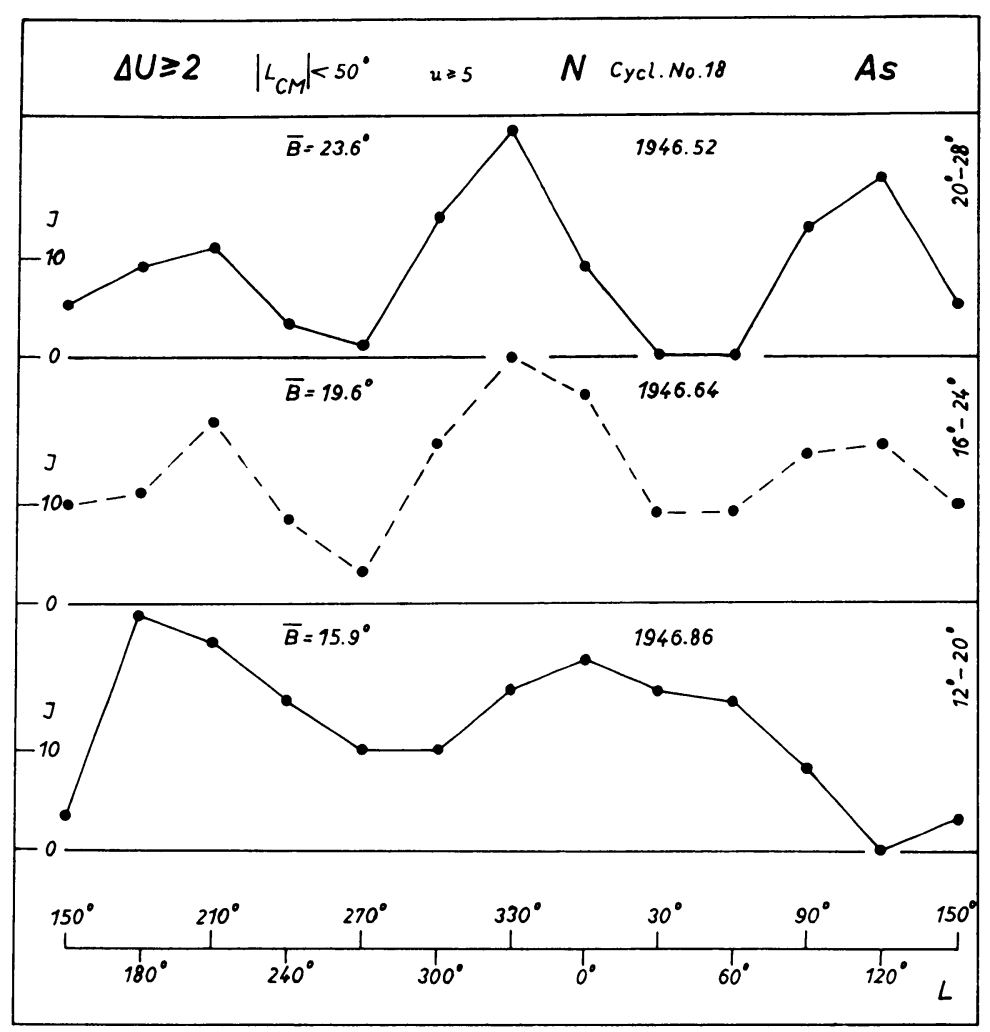

FIG. 3. Examples of distribution of sunspot activity in longitude.

be more exact, there are some shifts in longitude also, but only comparatively small ones and in both directions.

\section{Migrations indicated by Active Regions}

The migrations of sunspot activity becomes even more apparent if we do not follow a strict statistical procedure, as in the above, but simply try to select the most important active regions. We chose $\Delta U \geqslant 5$ as a criterion of intense activity of a spot group. Thus we used only the spot groups that showed an umbral increase of at least 5 areal units from one day to the next within a distance of $60^{\circ}$ longitude from the central meridian. Here, however, we used all spot groups whose lifetimes were at least 3 days $(u \geqslant 3)$.

In the light of what we outlined in the foregoing section, it was not too difficult to pick out those active regions that are probably members of tracks which come into being through migrations of sunspot activity. In Figure 4 we show for two periods of 
approximately 1-year possible migrations of this kind, indicated by active regions of the Southern solar hemisphere.

The latitude and longitude variation of about a dozen tracks of solar active regions are plotted in Figure 4. The dots generally represent three and more spot groups (in some cases even seven) and in most cases $\Sigma \Delta U>100$, the given positions of the regions being moreover mostly comparatively quite close to the actual measured position of the spot group. The related differences in latitudes and longitudes are nearly always less than $5^{\circ}$ and $40^{\circ}$ respectively. The times of observations that relate to the dots are all within three solar rotations approximately.

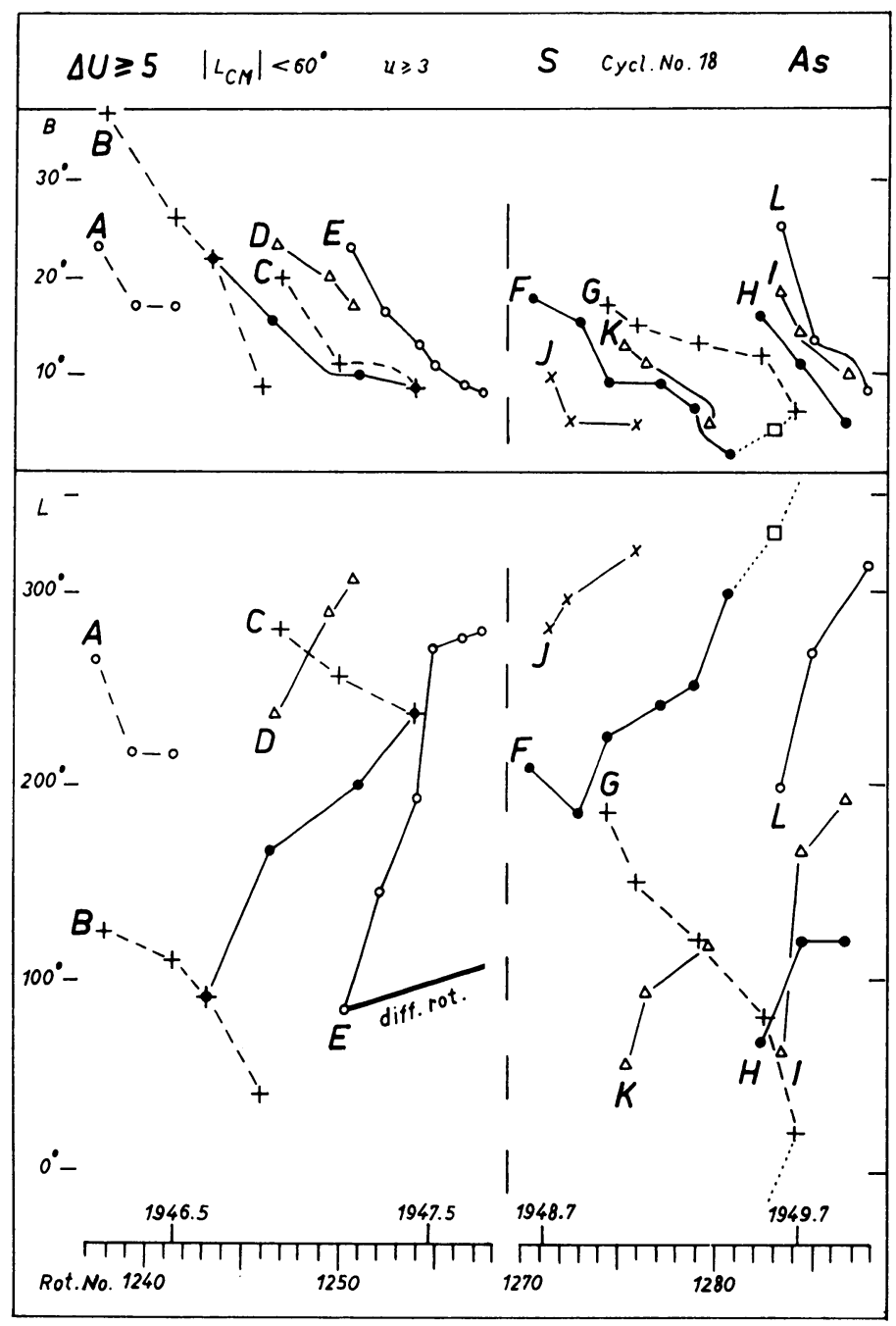

FIG. 4. Coordinates of solar active regions. 
The examples in Figure 4 represent 70 and 105 spot groups, i.e., $29 \%$ and $48 \%$ of those used, from the years 1946-47 and 1948-49 respectively. But we should point out that the corresponding percentages of the $\Sigma \Delta U$ values are 46 and 57 , i.e., quite high. Accordingly it is justified to suspect that our samples in Figure 4, from the Southern hemisphere and the periods indicated, show in fact one of the main characteristics of sunspot activity.

\section{Conclusions}

Summing up what has been outlined, it may be taken as a fact that solar activity has a rapid variation in latitude. It is easy to see that one should conclude from this fact that the butterfly diagram is mere consequence of the latitude variation of migration in sunspot activity. It is therefore a prime necessity that this rapid migration should be accounted for by the theories. The velocity of the migrations in question has by no means a constant value, though nevertheless we find it very frequent for $1.5^{\circ}$ per rot.

The speed of the shift, as well as its direction in longitude, of the migrations of sunspot activity seems to be even more variable than is the case with latitude variations. It is very important to note, however, that the differential rotation cannot be the reason for these displacements in longitude. (As an example, our calculations in Figure 4 show the shift that could have originated in differential rotation in the case of track E.) From this property alone, it follows that the disturbances which are responsible for the formation of sunspots must migrate quite deep-seatedly under the photosphere. Consequently the final cause of the sunspot phenomenon can hardly be considered a process that takes places on the solar surface alone.

\section{Acknowledgements}

We are indebted first of all to Miss E. Horváth and also to Miss Gy. Gyertyános, who compiled most of the necessary data. Our thanks are also due to Dr.I. Guman and Mr.I. Ökrös, who actively participated in the first preliminary phase of this investigation.

\section{References}

Bell, B. (1960) 'On the Structure of the Sunspot Zone', Smithson. Contr. Astrophys., 5.

Dezső, L. (1964) 'Statistical Investigations of Sunspots by a New Method', Publ. Debrecen Obs., 1. Kuleshova, K. F. (1962) 'The Fine Structure of the Spot-Formation Zones', Astr. Zu., 39, 273-277 (=Soviet Astr. - AJ., 6, 213-216)

Lockyer, W.J.S. (1904) 'Sunspot Variation in Latitude 1861-1902', Proc. Roy. Soc., 73, 142-152. 